\title{
Creating a compelling business case for employers; psychosocial benefit for mature workers
}

Kristine Gatt

WCD Workers' Compensation Solutions, Hawthorn VIC 3122, Australia. kgatt@wcdcomp.com.au

Twenty years ago, those of us in the allied health field were trained to believe that mature people didn't lodge workers' compensation claims and mature workers didn't have injuries because they 'knew their work environment so well'. Only 7 years ago, insurers believed that their book of claims in Workers' Compensation had no age related correlation to claims costs. We now know that all of these assumptions do not stand up in relation to the current workforce in Australia and we are building a wealth of knowledge about the mature cohort in today's workforce.

This presentation will guide the audience through the process of developing a compelling business case to assist employers understand the cost benefit of proactively engaging with their mature employees and the benefits for both the workers over fifty five years of age and businesses in Australia.

In developing a business case, the first stage of a strategy is being formulated for the care and well-being of mature workers, this leads to preparedness for effective return to work measures in the event of injury and health maintenance strategies for mature workers. The side benefits to a business are vast; from reduced turnover and associated costs, the retention of knowledge to reduced insurance costs and productivity benefits.

Five years of analytical data will be presented to demonstrate the trending, the influences and precautionary tales for those committed to the psychological well-being of the mature workforce in Australia. 